\title{
Etude de l'altération de la calcarénite des monuments de Rabat: Influence de la Pollution Atmosphérique et des Aérosols Marins
}

\author{
ZAOUIA Nouhad $^{1}$, EL WARTITI Mohamed ${ }^{1},{\text { NAHRAOUI Fatima } \text { Zahra }^{1} \text { et DABI Sana }}^{1}$ \\ ${ }^{1}$ Laboratoire de Géologie Appliquée: Ressources minières, ressources en Eau, Patrimoine géologique et Environnement. \\ Faculté des Sciences de Rabat, Université Mohammed V- Agdal, Maroc.
}

\begin{abstract}
Résumé. Le Maroc par sa diversité culturelle à travers les âges, est doté d'une panoplie de villes impériales reconnues à l'échelle mondiale. Rabat la capitale actuelle du royaume, inscrite au patrimoine mondial de l'UNESCO en 2012, apporte le témoignage architectural des différentes dynasties qui s'y sont succédé depuis les romains. Ces édifices monumentaux principalement construits à base de calacarénite souffrent depuis quelques décennies d'une accélération potentielle de leur dégradation à cause de l'influence de conditions environnementales de plus en plus agressives.

En effet, les monuments choisis pour cette étude; qui pour la plupart datent de l'époque prospère des Almohades au XII éme siècle; montrent une dégradation poussée se manifestant en surface sous forme de noircissements, de croûtes noires, d'alvéolisation ou d'efflorescences salines.

Afin de déterminer les causes et les processus de cette dégradation, nous avons effectué dans un premier lieu une étude géologique en vue de ressortir les caractéristiques pétrographiques de ce matériau et les formes d'altération qui l'affectent au niveau des carrières de la région, pour passer ensuite à la stratégie d'investigation qui s'appuie sur l'étude analytique des surfaces altérées, qui consiste en l'identification pétrographique, minéralogique et chimique des produits de l'altération, à l'aide de techniques de laboratoire telles que le microscope électronique à balayage équipé du système EDX, la diffraction aux rayons X, et l'analyse des sels solubles par la conductimètrie, la chromatographie ionique et la spectrométrie par flammes.

Le but du présent travail est de constituer une base de données pour orienter les recherches ultérieures sur la problématique de la conservation du patrimoine bâti et optimiser le choix des matériaux de remplacement, tout en prenant en compte tous les mécanismes et démarches nécessaires pour une meilleure protection dans le cadre d'un développement urbain en perpétuelle expansion.

Mots clés : Monuments, Altération, Calcarénite, Pollution Atmosphérique, Aérosols, Sels, Alvéolisation, croûtes noires, Etude analytique.
\end{abstract}

\section{Introduction}

Rabat, capitale actuelle du Royaume du Maroc, possède un patrimoine culturel riche par sa qualité, son abondance et l'étendue chronologique qu'il recouvre. La direction du patrimoine et des monuments historiques a toujours été attentive à préserver le mieux possible les témoins du passé. Néanmoins, le problème endommagement de ces prestigieux monuments persiste toujours. C'est dans cette optique de participation à la conservation, que $s$ 'est orientée notre recherche afin de permettre une prise de conscience sur la nécessité d'interventions multidisciplinaires, et le développement de techniques d'analyses et d'essais adaptés à l'étude des matériaux du patrimoine architectural.

Les monuments choisis pour cette étude datent pour la plupart de l'époque prospère des almohades (XIIème siècle). Ces vieilles constructions évoluent actuellement dans un environnement agressif, La contamination de l'air par des acides s'ajoute à la salinité naturelle d'une atmosphère océanique, pour induire des transformations dans le matériau calcarénitique, vulnérable par sa nature. C'est dans cette perspective que le présent travail est mené pour décrire et $d$ 'inventorier les différentes pathologies visibles dans un grand nombre de monuments de la ville, et $d$ 'essayer $d$ 'identifier les facteurs et mécanismes responsables. La stratégie d'investigation s'appuie sur une étude analytique des surfaces altérées, qui consiste à l'identification pétrographique, minéralogique et chimique des produits de l'altération, dans le but de constituer une base de données nécessaires pour orienter les 
recherches ultérieures sur la problématique de la conservation du patrimoine, dans le schéma d'un développement urbain en véritable expansion.

\section{Généralités sur la zone d'étude}

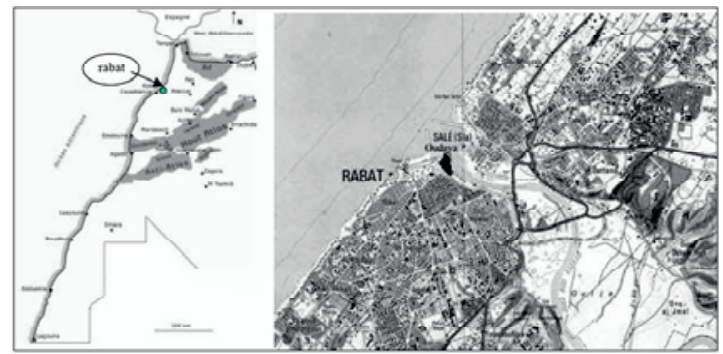

Fig.1: situation géographique de Rabat.

Rabat, la capitale du royaume depuis 1912, est située sur la rive estuarienne gauche du Bou Regreg, sur la côte atlantique marocaine entre la latitude nord $37^{\circ} 82$ et la longitude $10^{\circ} 19$ ouest de Greenwich (mesurée aux Oudayas). Située sur la rive gauche de l'embouchure d Bou Regreg, face à la ville de Salé «figure.1»; Cernée de remparts, la capitale du Royaume possède un important patrimoine architectural qui compte de nombreux monuments comme les portes de l'enceinte de la Kasbah des Oudayas, le minaret de la mosquée Hassan (XIIe siècle), qui s'élève à $55 \mathrm{~m}$, un des rares vestiges de cet édifice inachevé et, à proximité de la ville, la nécropole de Chellah.

La position géographique du Maroc fait qu'il se caractérise par un climat méditerranéen avec deux saisons bien marquées, un été chaud et sec et un hiver humide avec des précipitations irrégulières. Du point de vue climatique Rabat par sa situation atlantique subit toutefois des influences océaniques qui se traduisent par un adoucissement des températures, une réduction des écarts thermiques et une humidité de l'air qui diminue en s'éloignant du littoral; avec un été chaud où les maxima atteignent les $26^{\circ} \mathrm{C}$ et les minima les $17^{\circ} \mathrm{C}$. C'est la saison la plus chaude et la plus sèche. Pour la pierre des monuments $c^{\prime}$ est la saison de l'évaporation de l'humidité accumulée dans la roche, qui s'accompagne notamment par des en surface ou subsurface, de matières minérales plus ou moins cristallisées. Il s'avère aussi que les conditions alarmantes de pollution atmosphérique par émissions des automobiles, particulièrement les taux élevés en dioxyde de soufre, sont favorisantes de l'altération des monuments. La pierre calcaire poreuse constitue un système ouvert aux polluants, d'autant plus susceptibles d'être agressifs dans le climat littoral sub-humide de la ville, catalyseur des interactions polluant acide/roche calcaire. Les polluants artificiels qui jouent un rôle dans la détérioration de la pierre sont les composés sulfureux (dioxyde de soufre, sulfure d'hydrogène, le fluorure d'hydrogène, aérosols sulfatés), les oxydes d'azote, l'ozone, le gaz carbonique. Toutefois le SO2 est le principal polluant qui intervienne dans la dégradation des pierres. En effet, les dépôts sombres et les croûtes noires fréquemment observées à la surface des monuments sont essentiellement composés de sulfate de calcium qui est formé par la réaction entre le dioxyde de soufre et le carbonate de calcium des pierres calcaires

\section{Méthodologie du travail}

Pour mener ce travail à terme, deux approches ont été utilisées: Un travail de terrain basé sur l'observation et l'échantillonnage au niveau des carrières dont serait issue la calcarénite en œuvre dans les monuments concernés par cette étude et un travail de laboratoire consacré à l'application de techniques analytiques les plus fréquemment utilisées.

Le travail de terrain englobe l'étude géologique des carrières qui va nous permettre permet une compréhension de l'évolution de la calcarénite dans son milieu naturel, suivit par l'étude des monuments et l'altération de la calcarénite en œuvre.

Le travail de laboratoire consiste à appliquer quelques techniques d'observation ou d'analyse sur les échantillons de roche des carrières et ceux des monuments, dans le but de comparer la roche altérée à la roche saine, et de déterminer par des méthodes qualitatives les produits de 1'altération, en adoptant des techniques d'analyse minéralogiques ( $\mathrm{La}$ microscopie optique, $\mathrm{La}$ microscopie Electronique à Balayage (MEB), Diffraction des rayons X sur poudre) et des analyses chimiques (La Chromatographie Ionique (CI), La Spectrométrie d'Absorption Atomique (SAA), La conductimètrie)

\section{Les calcarénites des carrières de Bouknadel}

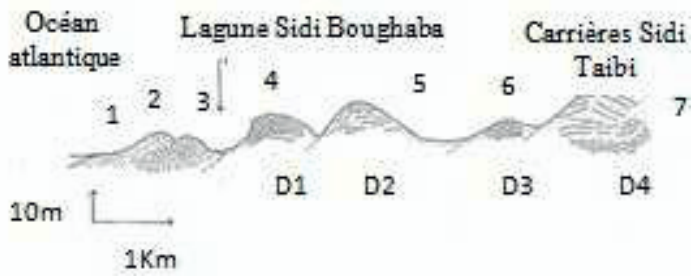

Fig.2: Coupe transversale à $1 \mathrm{~km}$ au sud de Mehdia [1]. 1: plage; 2: Dune blanche; 3: Dune grise; 4: complexe dunaire consolidé D1; 5: Complexe dunaire consolidé; D2; 6: Grès dunaire lapiazé localement altéré; 7: Complexe dunaire consolidé D4. 
Le cordon littoral interne D4 «figure. 2 », situé le long de la route principale $\mathrm{n}^{\circ} 2$, à $1^{\circ}$ Est, comporte les formations de calcarénite les plus consolidées. C'est dans cette dune que sont ouvertes actuellement un grand nombre de carrières $d$ 'exploitation de géomatériaux, dont les carrières de BouKnadel qui livrent notamment la calcarénite ornementale et la pierre de taille, les plus sollicitées des artisans de la pierre. L'étude de cette calcarénite montre un fond commun d'éléments figurés. Elles sont constituées par deux fractions détritiques: une fraction bioclastique et une fraction terrigène avec un ciment et/ou une matrice carbonatés « figure. 3 ».

L'observation de l'échantillon $\mathrm{BKCr}$ au MEB équipé de $\mathrm{EDAX}$, montre une micromorphologie zonaire avec une alternance de plages ferrugineuses denses et de plages carbonatées relativement poreuses « figure. 4 ».

L'étude de la diagenèse montre son influence directe sur la porosité et le degré de consolidation des calcarénites de la région; on observe deux types de diagenèse: une diagenèse précoce en milieu marin peu profond, généralement en zone vadose marine (intertidal à supratidal), comme en témoigne la présence d'une mince enveloppe micritique et des cristaux fibreux à microstalactitiques autour des éléments figurés et une diagenèse tardive en zone vadose météor ique (milieu continental), se manifestant par la précipitation des cristaux microsparitiques à sparitiques de formes variées. La diagenèse se fait par cimentation pédogénétique par la calcite micritique en auréole des éléments figurés., cimentation par de la microsparite à sparite

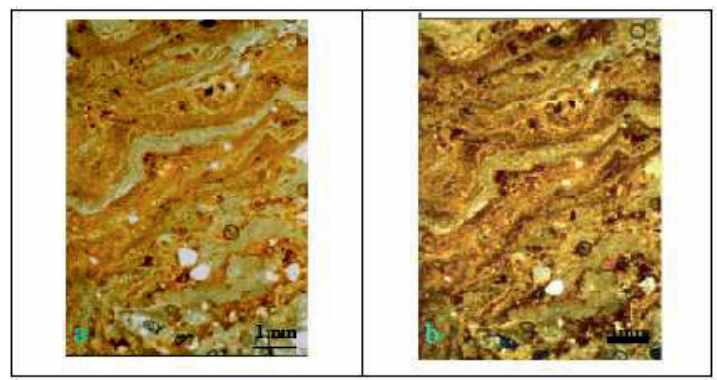

Fig.3: Micromorphologie de la croûte calcaire de la coupe Bouknadel A montrant l'alternance de lamines claires carbonatées et de lamines ferrugineuses au microscope optique, $\mathrm{a}$ : en LN et $\mathrm{b}$ : en LP.

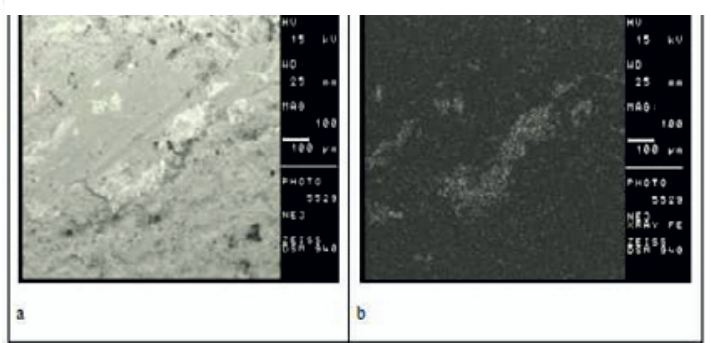

Fig.4:Image M.E.B. de la croûte laminaire de la limite (poche de dissolution-roche) ; (a : micromorphologie laminaire de bandes claires et de bandes chromatiques ; $\mathrm{b}$ : Analyse aux rayons X de Fe par MEB sur la prise de vue (a) montrant 1'occurrence de plages de fer qui imprègne les lamines chromatiques). drusique dans les espaces et par épigénisation des minéraux primaires dans les niveaux encroûtés et une précipitation de calcites en aiguilles (lublinite) dans la porosité de la croûte calcaire, témoignent $d$ 'une altération parfois récente ou active.

Les essais géotechniques effectués sur notre calcarénite ont montré qu'elle a des valeurs de résistance à la fragmentation qui sont très élevées, des valeurs de résistance à l'usure (M.D.E) qui sont également très élevées, l'essai de la résistance à la rupture testé par l'indice de résistance (IR) montre que les calcarénites plioquaternaires du secteur d'étude résistent peu à la rupture avec des valeurs comprises entre 0.01 et $0.84 \mathrm{KN} / \mathrm{cm} 2$ et une valeur moyenne de $1^{\prime}$ ordre de $0.5 \mathrm{KN} / \mathrm{cm} 2$, les calcarénites étudiées se révèlent peu denses puisque dans les carrières de Bouknadel la densité apparente varie de 1.39 $\mathrm{g} / \mathrm{cm} 3$ à $1.47 \mathrm{~g} / \mathrm{cm} 3$ et l'indice $\mathrm{n}$ de porosité montre que ces roches consolidées sont moyennement poreuses avec des pourcentages de porosité compris entre $45.14 \%$ à $47.29 \%$ à Bouknadel.

Les caractères pétrographiques de la calcarénite ont une influence directe sur les propriétés géotechniques de cette dernière: La richesse en bioclastes, la diagenèse en général, et la dissolution en particulier, qui affectent ces matériaux déterminent le taux de porosité intragranulaire et donc la dureté du matériau, Le pourcentage du ciment carbonaté qui est relativement faible dans les calcarénites dunaires $(30 \%)$ et plus important (jusqu'à $80 \%$ ) dans les calcarénites marines où la cimentation est précoce, joue un rôle important dans la formation de la porosité intergranulaire et dans la détermination de la rigidité de la roche et enfin les calcarénites étudiées sont en général des grainstones à ciment microsparitique à sparitique et pauvre en micrite, et donc, à résistance faible à moyenne.

\section{Morphologie et analyses des altérations dans les monuments}

La conservation des monuments historiques nécessite de nombreuses études qui ont permis de référencer les formes d'altération les plus fréquentes qui peuvent contribuer à la dégradation de la calcarénite de ces édifices. Les travaux de Jeanette [3], ont fourni une classification des plus employées parmi les spécialistes de la pathologie de la pierre, qui se base sur le type de processus d'altération : physique, chimique, ou biologique.

En appliquant cette classification à Rabat nous avons pu avoir un premier constat sur les types d'altérations et dégradations qui affectent les monuments de cette ville et qui peuvent êtres décrites comme suit: Les Altérations physiques qui sont dues à une simple érosion mécanique de la roche, leur intensité dépend de la turbulence du 
ruissellement [2] et comprennent les dissolutions et ravinements, et les fissures. Les altérations chimiques «figure. 5 », « figure. 6 » qui nécessitent 1'intervention de 1'eau liquide [4]. Cet agent de 1'altération est fourni à Rabat par les eaux de pluies, les précipitations occultes et 1'humidité ascensionnelle dans le monument, ces altération se manifestent sous forme de patines «figure. 8 », noircissement et croûtes noires, arénisations et les alvéolisations «figure. 5 », «figure.7» et d'efflorescences (dépôt de sels solubles). [5]

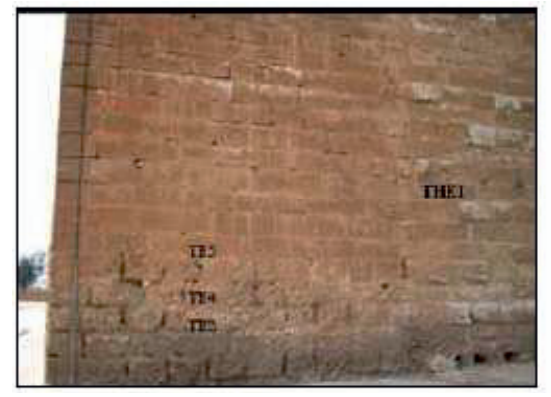

Fig.5 : façade Est de la Tour Hassan montrant la dégradation par remontée capillaire à la base $d u$ mur et le noircissement de la partie médiane et 1'emplacement des échantillons TH1, TH3, TH4 et TH5.

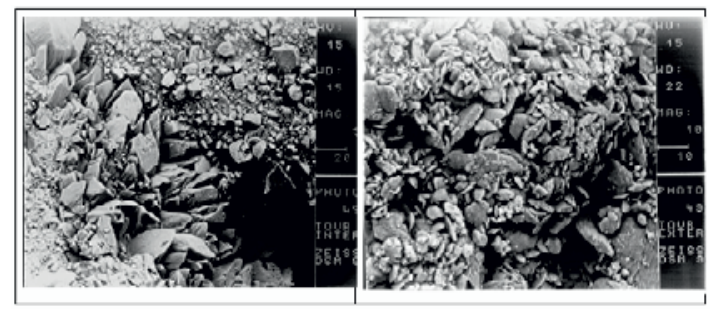

Fig.6 : (a) : Microphotographie de l'échantillon de calcarénite (TH1int) de la Tour Hassan vu au MEB ; face interne peu altérée de l'échantillon, les cristaux de calcite (a) sont pris dans un ciment microspathique qui confère à la roche une certaine cristallinité ; (b) : Microphotographie de l'échantillon de calcarénite (TH1ext) de la Tour Hassan vu au MEB. Face externe altérée de l'échantillon. Les cristaux de calcite semblent libérés et le ciment dissous ce qui augmente la porosité de la roche et peut entraîner sa désagrégation.
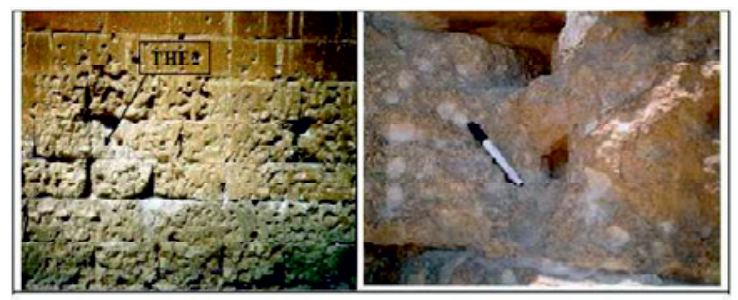

Fig.7 : Alvéolisation très intense dans le bas du mur est de la Tour Hassan.

Finalement nous avons la biodégradation
« figure.9 » qui est dû à l'action des êtres vivants et qui affecte largement les monuments étudiés, notamment les façades nord à nord-ouest, à cause des vents chargés d'humidité et parfois de matière vivante. S'ajoute à ceci la restauration inadaptée de ces monument en utilisant le ciment moderne qui est nocif, car il est très étanche et empêche l'évaporation à travers le joint et celleci se fait, donc, via la pierre qui se dégrade relativement plus vite « figure.10». [5]

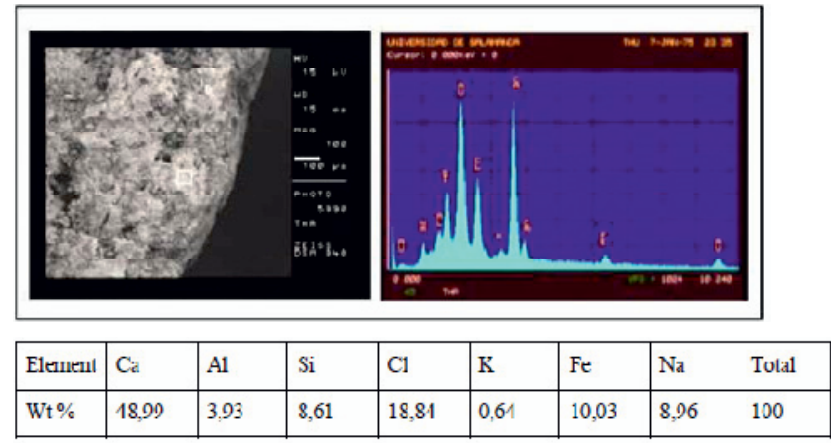

Fig.8 : a/microphoto au MEB d'une section transversale de la patine ocre THS, b/microanalyses chimiques de la zone encadrée; résultats des microanalyses chimiques de la zone encadrée de la façade Est de la tour Hassan.

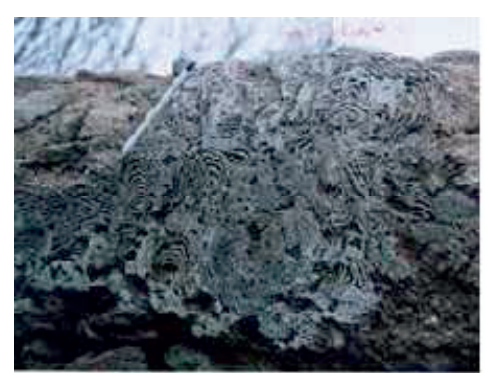

Fig.9 : Biodégradation par des mousses sur un rocher de calacrénite aux Oudayas.

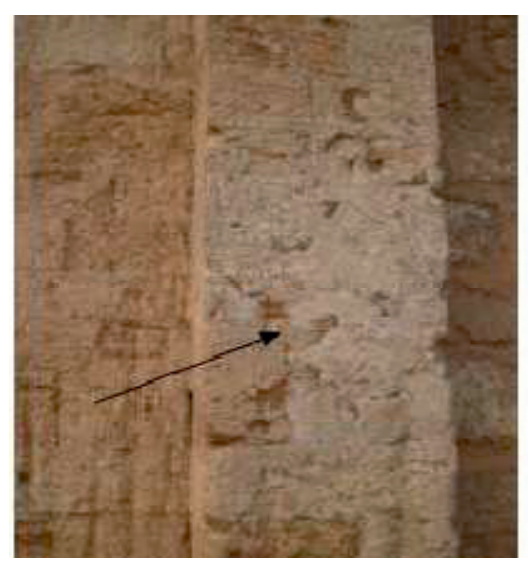

Fig.10 : Colmatage inapproprié des zones érodées par du ciment. 


\section{Résultats et Discussion}

On se basant sur l'étude de la morphologie et les analyses des altérations dans les monuments de Rabat, nous avons pu définir les principaux facteurs régissant l'altération de ces édifices historique. Ces facteurs sont ceux susceptibles d'induire ou favoriser une modification minérale ou texturale dans les calcarénites. Ces facteurs peuvent être liés à la nature lithologique de la roche et dans ce cas ils sont intrinsèques, ou ils peuvent être liés aux paramètres du milieu environnant, régional ou local, et influencer son état de conservation, et être considérés comme des facteurs extrinsèques. Ces facteurs sont généralement les paramètres du milieu qui règnent autour du monument, l'humidité, l'eau de pluie, les variations de températures et l'insolation, le vent, la contamination par les apports atmosphériques, et Les aérosols marins.

Les observations visuelles et l'étude analytique avec des méthodes qualitatives permettent l'identification des facteurs et processus probables de l'altération de la calcarénite des monuments étudiés, liés à l'influence d'un climat littoral dans une région au caractère urbain.. Le noircissement et les croûtes superficiels sont les manifestations les plus fréquentes de la dégradation par sulfatation de la roche fortement carbonatée. Le rôle joué par le $\mathrm{SO} 2$ de 1'air est manifeste. Il a permis, avec $1^{\prime}$ expansion notoire du parc automobile de la ville, l'accélération de la dégradation de la pierre en seulement quelques décennies, les variations dans l'intensité de ce type d'altération sont contrôlées par le degré d'exposition directe aux pluies. L'implication du climat marin est aussi évidente. La quantité des chlorures de sodium et leur fréquence dans les sites appréhendés sont d'une importance considérable, traduisant un grand apport d'origine marine à la roche soumise à 1'action des aérosols marins. Ceci se traduit par des dégradations par arénisation, pulvérulence et alvéolisation de la pierre, et parfois un net recul de la surface., aux quels s'ajoute la dégradation biogénique. Ces facteurs locaux sont actifs grâce à la vulnérabilité de la calcarénite, induite par ses faibles propriétés géotechniques. [5]

\section{Conclusion et Perspectives}

Les altérations identifiées sur les monuments de Rabat étudiés, présentent des morphologies différentes, à savoir, le noircissement, les croûtes noires, les patines ocres, l'arénisation, l'alvéolisation et la biodégradation. Elles sont inhérentes à la nature calcaire, et la texture très poreuse de la calcarénite en Euvre. L'examen au microscope électronique à balayage, de la pierre altérée, montre la concentration des dommages sur la partie superficielle, attestant de l'influence des paramètres atmosphériques dans la dégradation de la pierre. La pollution atmosphérique (pluies acides, mais aussi SO2 de l'air et dépôts secs de particules de sulfates, suies et hydrocarbures), est un facteur aggravant de la dégradation naturelle subie par la calcarénite mise en Euvre depuis des siècles, dans une atmosphère à influence marine. L'établissement d'une modélisation de l'impact de la pollution atmosphérique, et des effets de l'atmosphère marine, sont les étapes futures dans la compréhension de la problématique de l'altération des monuments de Rabat. Dans le domaine de la restauration, ces travaux rendraient possible un meilleur choix des matériaux de remplacement. Prendre en compte tous les mécanismes qui interfèrent est utile à la réalisation d'une bonne protection. [5]

\section{Références bibliographiques}

1. M. ABERKAN, Etude des formations quaternaires des marges du Bassin du Rharb (Maroc Nord occidental). Thèse d'Etat, 290p., Univ. Bordeaux I (1989).

2. D. CAMUFFO, Physical weathering of stones. The Science of the Total Environment, vol. 167, pp.1-14 (1995).

3. D. JEANNETTE, Morphologie et nomenclature des altérations. In : la conservation de la pierre monumentale en France, les Presses du CNRS, éd., Paris, J. Philippon, D. Jeannette et R. A. Lefèvre, coordinateurs, 51-72 (1992).

4. G. PEDRO, Un phénomène normal de la surface de la planète : L'altération des roches. Actas del workshop Alteracion de granitos y rochas afines, empleados como materiales de construccion. Ed. M. A. Vicente Hernandez, E. Molina Ballesteros, V. Rives Arnau ; pp.17-50. CSIC Madrid (1993).

5. N. ZAOUIA, Contribution à l'étude de l'altération de la calcarénite en œuvre dans les monuments de Rabat Influence de la pollution atmosphérique et les aérosols marins. Thèse de doctorat, 172p., Univ. Mohammed V (2007) ; 